\title{
PAPER
}

\section{Acoustical analysis on the sawari tone of Chikuzen biwa}

\author{
Tomoyasu Taguti ${ }^{1}$ and Yoshimasa (Kakuryo) Tohnai ${ }^{2}$ \\ ${ }^{1}$ Faculty of Science, Konan University, \\ 8-9-1 Higashinada, Kobe, 658-8501 Japan \\ e-mail:taguti@konan-u.ac.jp \\ ${ }^{2}$ Buzan Senior High School, Nihon University, \\ 5-40-10 Otsuka, Bunkyo-ku, Tokyo, 112-0012 Japan
}

( Received 8 May 2000, Accepted for publication 24 October 2000 )

\begin{abstract}
The sawari is an instrumental mechanism of a certain class of stringed instruments so that the string touches to it repeatedly when vibrating. The Chikuzen biwa is one of Japanese plucked stringed instruments; it is equipped with a sawari which is a narrow strip of surface on the top of shirabeguchi (the nut). It is known that only a minute change in the shape of this surface results in a large difference in the quality of the resulting "sawari" tone. This paper studies the sawari tone under different grades, or strengths, of the sawari created by shaving the top surface of shirabeguchi differently with masterly craftsmanship, together with one without sawari (no shaving at all), using an excellent Chikuzen 5-stringed biwa, to compare quantitatively the effect of the degree of shaving on the resulting sound. The analysis shows the temporal development of the amplitudes of up to 24th partials for open strings under each of the above-mentioned sawari conditions. The sawari effect appears in two aspects: (1) to intensify the partials of 6th to 20th and up, and (2) to elongate their durations.
\end{abstract}

Keywords: Biwa, Sawari mechanism, Partials, Time-frequency domain

PACS number: $43.75 . \mathrm{Gh}$

\section{INTRODUCTION}

The biwa and shamisen, which are Japanese stringed instruments played with bachi (plectrum), are characterized in that they are equipped with an instrumental mechanism called sawari (touch). It is a mechanism to which the string touches repeatedly when vibrating.

The sawari of shamisen is rather well known to public [1]. It is provided for the first string only. Its structure is a narrow groove of a few millimeters in width, together with a pair of edges, settled just below and perpendicularly to the kamikoma (nut) where the second and third strings are laid. The first string is not on the kamikoma but laid across over the groove so that the edge at peg side works as the fixed end while the other edge is the point the string touches to and separate from when it vibrates. By this mechanism, the motion of the open first string, which otherwise would vibrate harmonically, gets vibrating components that are in high frequency region and are possibly non-periodic.

The sawari of biwa is provided for all strings. In the case of Chikuzen biwa, its structure is a narrow strip of surface, a few millimeters in width, created on the shirabeguchi (nut) which is made of susutake (tarred bamboo). It is created by shaving process so that the string touches to the surface nearly flat. The edge at peg side of this surface actually works as the fixed end so that the string touches to and separate from this surface when it vibrates. By this mechanism, the motion of open string gets vibrating components in high frequency region, and possibly non-periodic, similarly to the case of the open first string of shamisen.

Further, the biwas have wide, tall frets. By practice, stopping a string is made with a depression of string in between two frets by fingertip, but not on a fret. So, the vibration of stopped string at any fret gets the effect of sawari due to the surface of the fret at which the string is stopped. This is yet another point that differs from the shamisen.

Ando [2-5] has conducted acoustical studies on the Chikuzen biwa since mid 1970's. Tohnai-Kishi [6] and Kishi-Tohnai-Yamada [7] reported an acoustical analysis of Satsuma biwa. Tohnai has made a historical and structural study on Japanese modern biwas by conducting a field work of inspecting some 300 pieces of biwas [8], and also investigated the structures of sawari [9]. 


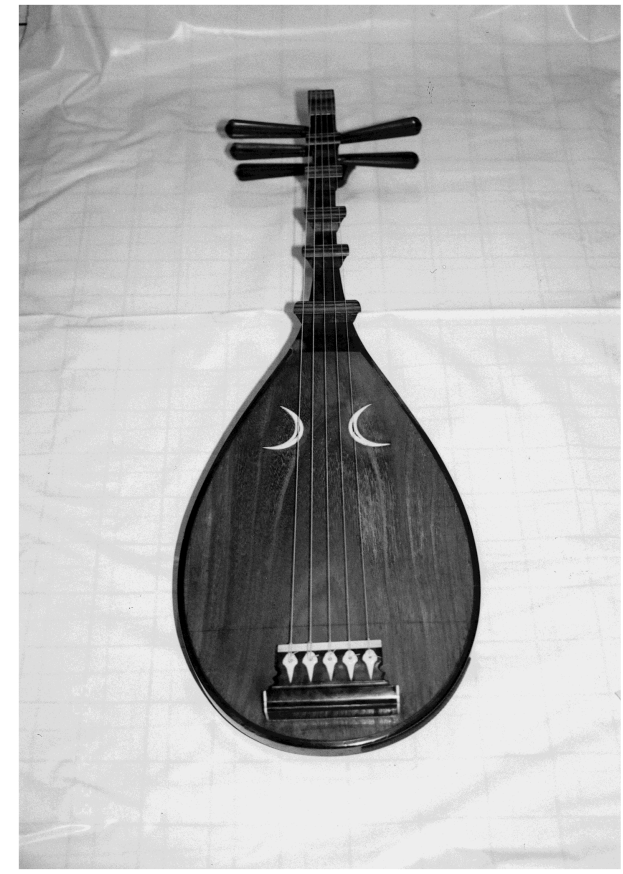

Fig. 1 Chikuzen 5-stringed biwa made by Chinsuke Tsurusaki. The body and top plate, respectively, are made of mulberry and paulownia; length $88.0 \mathrm{~cm}$ and width $32.5 \mathrm{~cm}$.

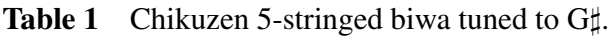

\begin{tabular}{lc}
\hline string & pitch \\
\hline First $^{\dagger}$ & C3 $\sharp$ \\
Second & G2 $\sharp$ \\
Third $^{\dagger}$ & C3 $\sharp$ \\
Fourth & D3 $\sharp$ \\
Fifth & G3 \\
\hline
\end{tabular}

${ }^{\dagger}$ First and third strings are unison.

Table 2 Four grades S0 S3 of sawari.

\begin{tabular}{ccc}
\hline grade & width $^{\dagger}$ & sawari effect \\
\hline S0 & $0 \quad \mathrm{~mm}$ & none \\
S1 & $0.7 \mathrm{~mm}$ & slight \\
S2 & $1.5 \mathrm{~mm}$ & moderate \\
S3 & $3.0 \mathrm{~mm}$ & strong \\
\hline
\end{tabular}

${ }^{\dagger}$ Approximate size.

This paper studies the relation between the sawari structure and the resulting sawari tone of Chikuzen biwa experimentally. An excellent biwa had been selected for the experiment, and a block of susutake had been mounted to it in advance. Then, the plucked tones of the instrument were recorded in anechoic room for different grades, or strengths, of sawari where these grades of sawari were created on the site. Preparation of the in- strument and the creation of sawari was made by the second author, and the recording and acoustical analysis by the first author. Playing the instrument was made by an expert player of Chikuzen biwa.

\section{SOUND MATERIALS}

\subsection{The Instrument and Making of Sawari}

The instrument was a Chikuzen 5-stringed biwa made by Chinsuke Tsurusaki, a biwa maker who was active during the period of late Meiji to Taisho Eras (1900's 1920's). The instrument was tuned to 7-hon ( $\mathrm{G} \sharp$ on the second string). See Fig. 1 and Table 1 for the instrument and its tuning.

A block of susutake mounted as shirabeguchi was about 100 years old. The height of shirabeguchi was adjusted so that there was a clearance of $0.2 \sim 0.25 \mathrm{~cm}$ from the top surface of the first fret (its name is $m o k u, 2.6 \mathrm{~cm}$ high from neck) to the strings at rest. Then, three grades, or strengths, of sawari structure, labeled S1, S2, and S3, were realized by a gradual shaving of the same susutake one after another, as shown in Table 2, together with grade S0 which was the status before shaving, i.e., of no shaving at all. Here, S3 was the one which yields the strongest sawari effect within the tolerable range of sawari tone, resulting in a sawari tone of the best quality. Figure 2(A) shows the susutake before shaving (top) and the sawari surface processed to grade S2 (bottom), and Fig. 2(B) a schematic drawing of sawari and the related parts of the instrument.

As a general tendency, the wider the strip of sawari surface, the stronger the sawari effect. But the actual situation is highly dependent on very small deviations in the contacting angle of surface to string, as well as on very small irregularities of the sawari surface, to the accuracy of sub-millimeters. So, it is difficult to establish a precise relationship between the width of sawari surface and the strength of sawari effect. The grades of sawari in Table 2 were made with the musical judgment by the second author who is a player of Satsuma biwa and also has had an expert skill with long experience in restoration and adjustment of biwa instruments.

\subsection{Recording Condition}

Single, plucked tones of open strings were picked up by a pair of microphones as a usual stereophonic recording, and recorded into DAT, in anechoic room. Here, the microphones were set so that they were aiming at the top plate of the instrument at a distance of $1 \mathrm{~m}$. The sound pressure at pick-up point by microphone was $80 \mathrm{~dB}$ SPL or thereabouts. The recording equipment was as follows: Brüel \& Kjær's microphones (type 4190) with preamplifier (type 2669L) and dual-microphone supply 
(type 5935L), and sound level callibrator(type 4231); and Sony's DAT recorder (DTC 2000ES).

At first, three tones were recorded for each of 5 open strings at grade $\mathrm{S} 0$, then those at grade $\mathrm{S} 1, \ldots$, and so on. After finishing all the recordings, the best ones were selected as the sound materials, 20 samples in total, one for each of 20 combinations ( 5 strings $\times 4$ grades). These tones were natural, single biwa tones of open string, that is, plucked tones of a single open string under the condition that other 4 strings were left open.

\subsection{Signal Processing}

The recorded sounds in one fixed track (that of left microphone) were taken as the sound material for the subsequent processing. They were pre-processed with a high-pass filter of Butterworth's 4th-order with cut-off frequency $80 \mathrm{~Hz}$ to suppress low-frequency noises such as atmospheric pressure waves. The effect of filtering on the sound signals was that it reduced the amplitude of the fundamental frequency of the second open string, the lowest of all the partials of our biwa tones, by a nominal $0.5 \mathrm{~dB}$.

The $\mathrm{S} / \mathrm{N}$ ratio of the sound signals after this filtering showed about $60 \mathrm{~dB}$ at their peak levels. The discrete Fourier transform (DFT) was applied to these sound signals to get their power spectra, to which Takasawa's algorithm [10] was applied further to get the timeevolutionary profiles of partials. The algorithm is based on the theoretical estimation on the error that the DFT inherently contains as a formula of discrete approximation for the integral of Fourier transform. It gives correction terms on both the amplitudes and frequencies of line spectra, i.e., those of single harmonic components, from the result of DFT with a Hanning window. Since it requires a relatively small number of sample points to get an accurate result, it is suitable to get the dynamic behavior of partials.

\section{TIME-FREQUENCY DOMAIN ANALYSIS}

Figure 3 shows the waveforms of the open first string. The top two plates are those at grade S0, where the left and right ones (duration $=20 \mathrm{~ms}$ each), respectively, are at time sections in the beginning and after $200 \mathrm{~ms}$; and the bottom two plates are those at grade S3 at the corresponding time sections.

Oscillating components of high frequencies appear a little more in the waveform at grade S3 than S0 in the beginning. But a big difference is observed at time section $200 \mathrm{~ms}$ so that the waveform at grade S0 slips into a smooth one while the one at grade S3 contains more components in high frequency region. Further, the amplitude at grade $\mathrm{S} 3$ is larger than the one at grade $\mathrm{S} 0$.

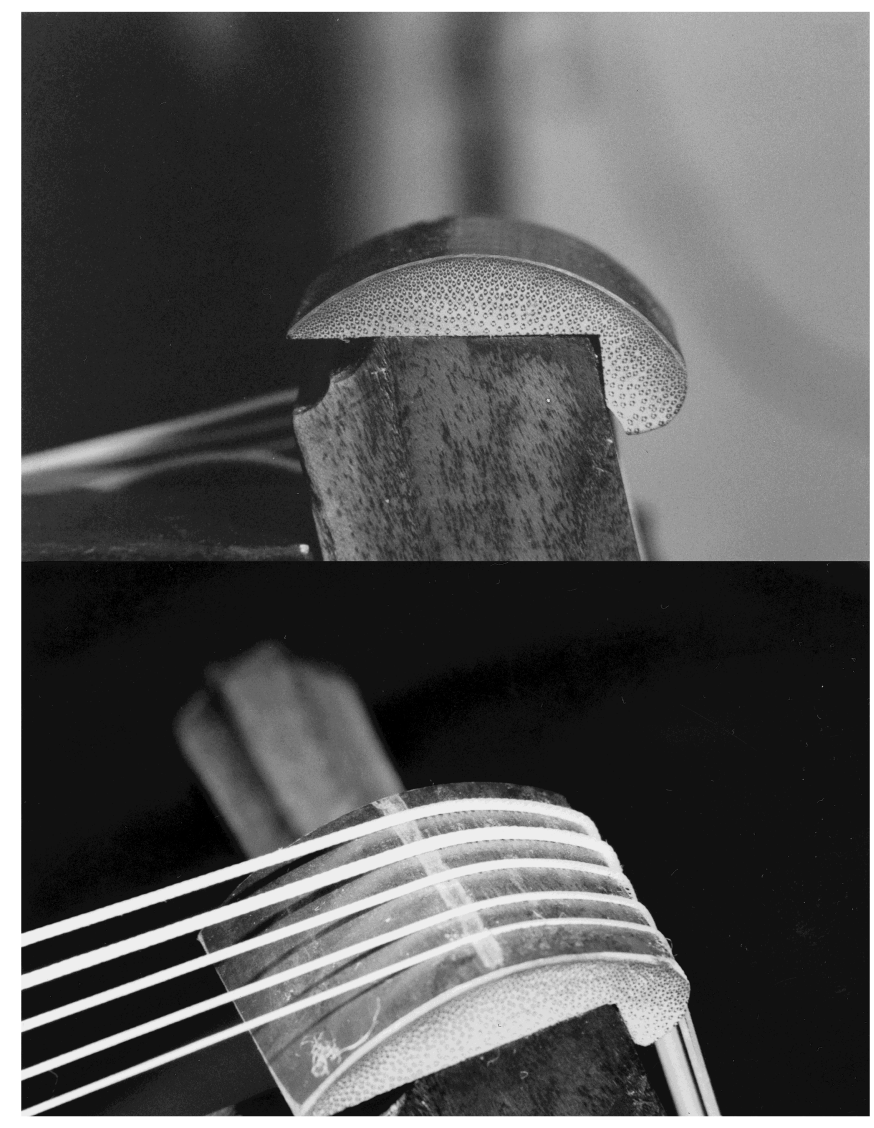

(A)

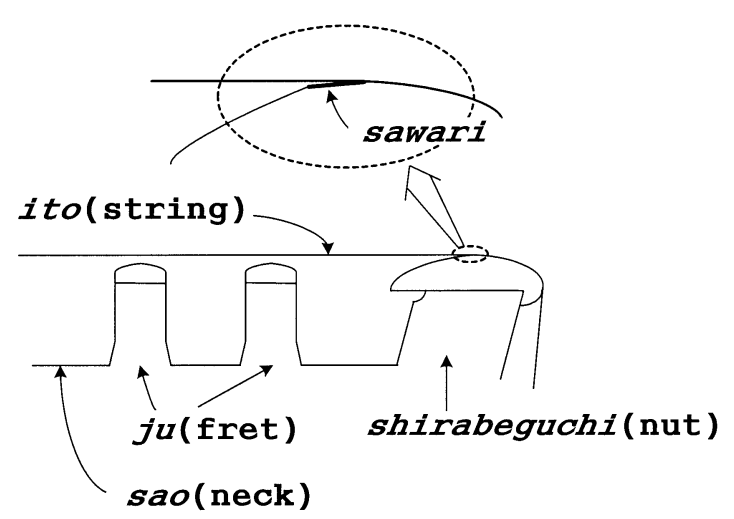

(B)

Fig. 2 (A) Susutake of shirabeguchi and making of sawari. Top: before processing, and bottom: processed to grade S2. (B) Sawari and the related parts of Chikuzen biwa. (Note. The slope of sawari is emphasized for illustration.)

Table 3 shows the temporal development of RMS (root-mean-square) value of waveforms S0 and S3 in the unit of $\mathrm{dB}$, computed with a $10 \mathrm{~ms}$ running window of integration, so that their peak values are adjusted to be $0 \mathrm{~dB}$ and to attain at time $0 \mathrm{~ms}$. The result implies that waveform S3 has a slower build-up of energy as well as a slower decay than S0. 


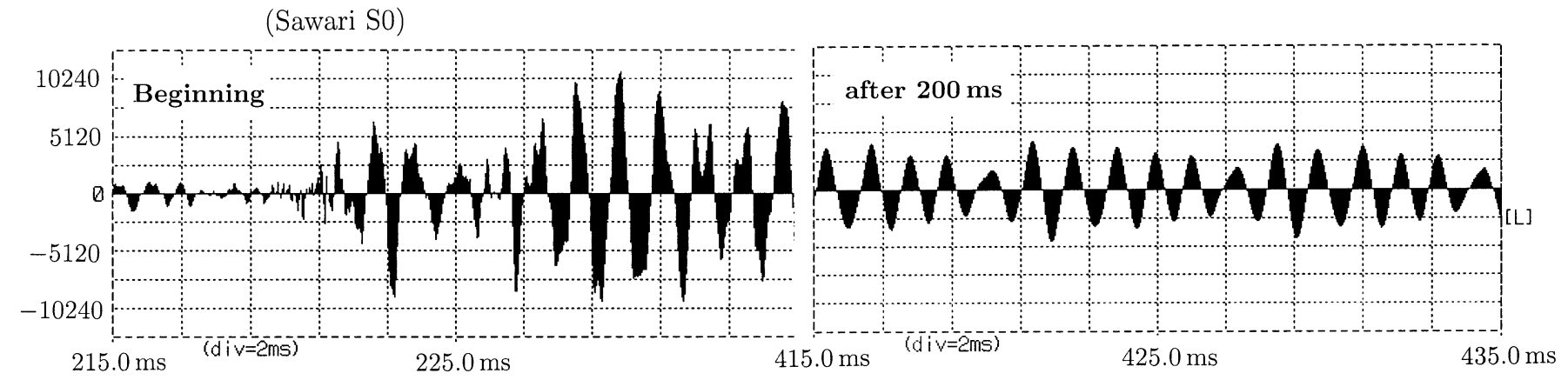

(Sawari S3)

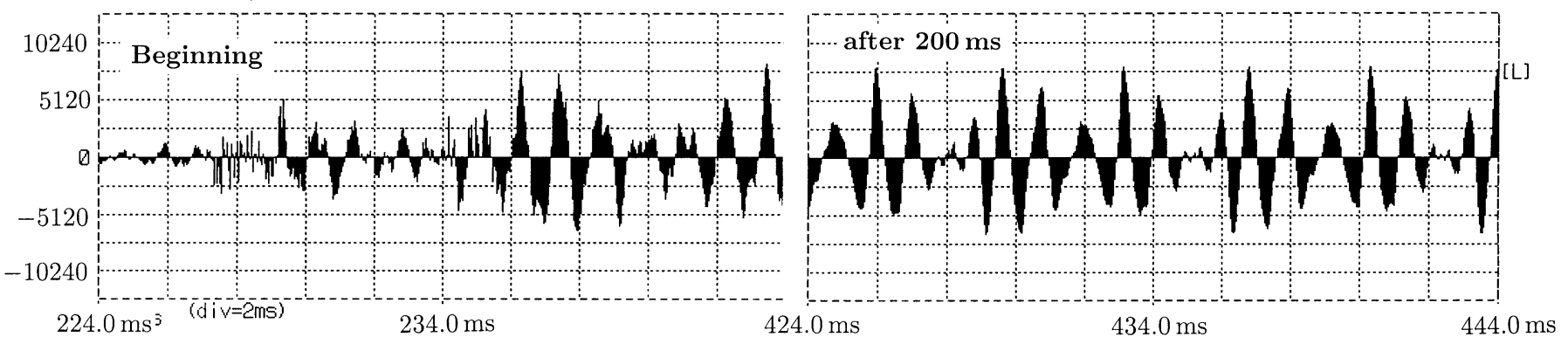

Fig. 3 Waveforms of the open first string; comparison of S0 (top) and S3 (bottom).

Table 3 Temporal development of RMS values $(\mathrm{dB})$ of waveforms $\mathrm{S} 0$ and $\mathrm{S} 3,1$ st string.

\begin{tabular}{ccc}
\hline \multirow{2}{*}{ time $(\mathrm{s})$} & \multicolumn{2}{c}{ RMS values $(\mathrm{dB})$} \\
\cline { 2 - 3 } & $\mathrm{S} 0$ & $\mathrm{~S} 3$ \\
\hline-0.05 & $*$ & -19.6 \\
-0.04 & -25.4 & -19.5 \\
-0.03 & -17.5 & -22.4 \\
-0.02 & -20.0 & -7.7 \\
-0.01 & -2.3 & -2.1 \\
\hline 0.00 & 0.0 & 0.0 \\
\hline 0.20 & -8.5 & -2.8 \\
0.40 & -10.6 & -1.3 \\
0.60 & -15.2 & -5.6 \\
0.80 & -21.2 & -9.8 \\
1.00 & -27.2 & -16.8 \\
1.20 & -34.8 & -26.6 \\
1.40 & -35.2 & -25.0 \\
1.60 & -40.2 & -31.9 \\
1.80 & -44.2 & -32.0 \\
2.00 & -47.1 & -34.4 \\
\hline 2.50 & -49.8 & -43.1 \\
3.00 & $*$ & -50.5 \\
\hline$*$ below $-55 \mathrm{~dB}$ & \\
\hline
\end{tabular}

Table 4 Spectral centroid $f_{\mathrm{c}}(\mathrm{Hz}), 1$ st string.

\begin{tabular}{cccc}
\hline S0 & S1 & S2 & S3 \\
\hline 864.1 & 968.9 & $1,147.1$ & $1,203.6$ \\
\hline
\end{tabular}

The DFT is applied to the interval of $341.3 \mathrm{~ms}(16,384$ points at $48 \mathrm{kHz}$ sampling, with Blackman's window) that starts at the time point when the acoustic energy reaches its maximal level right after onset. Figure 4 shows the power spectra for the open first string at grades $\mathrm{S} 0 \sim \mathrm{S} 3$. A clear distinction is seen that the partials of 7 th order and up get boosted as the sawari grade proceeds from S0 to $\mathrm{S} 3$.

Let us compute the spectral center of gravity, or spectral centroid, $f_{\mathrm{c}}$, defined by

$$
f_{\mathrm{c}}=\int_{f_{0}}^{f_{1}} f|F(f)| d f / \int_{f_{0}}^{f_{1}}|F(f)| d f
$$

where $|F(f)|$ is the power spectrum defined over the concerned interval of frequency $\left[f_{0} . f_{1}\right]$. Table 4 lists $f_{\mathrm{c}}$ 's correponding to the power spectra of $\mathrm{S} 0, \mathrm{~S} 1, \mathrm{~S} 2$, and S3 in Fig. 4, where $f_{0}=65.4 \mathrm{~Hz}$ (pitch C2) and $f_{1}=8,372.0 \mathrm{~Hz}$ (pitch C9). The $f_{\mathrm{c}}$ tends to be higher as the sawari grade proceeds, e.g., $f_{\mathrm{c}}$ of $\mathrm{S} 3$ is $1,203.6 \mathrm{~Hz}$, which is $339.5 \mathrm{~Hz}$ as high as that of S0.

Next let us examine the duration of tones by component-wise extraction of their partials. For this purpose we use Takasawa's algorithm as mentioned in Section 2 because it requires only a small number of sample points for DFT due to its high-precision formula for frequency/amplitude estimation. A time-window of 1,024 points $(21.3 \mathrm{~ms})$ was found to be suitable by experiment to extract the partials of the open first string (C $3 \sharp$, $138.6 \mathrm{~Hz}$ ). Figure 5 shows the temporal development of amplitudes of up to 24th partials at grades S0 and S3. 


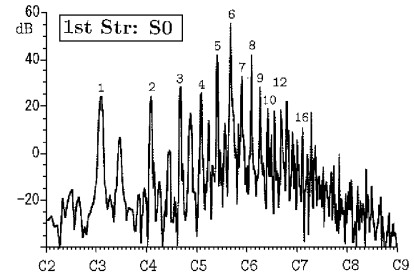

(a)

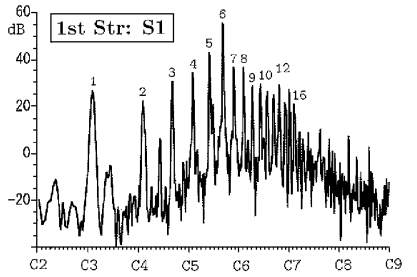

(b)

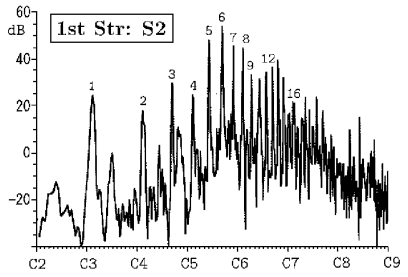

(c)

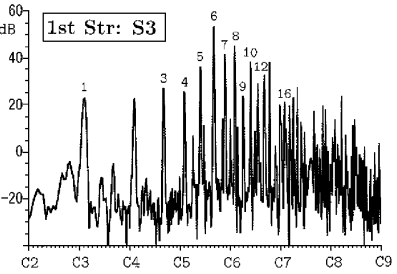

(d)

Fig. 4 Power spectra of the open first string; comparison of S0 S3. Results by DFT with Blackman's window for the interval of $341.3 \mathrm{~ms}$ (16,384 points) starting from the time when the acoustic energy reaches its peak level. The $x$-axis denotes the frequency in logarithmic scale marked with pitch names of $\mathrm{C}(\mathrm{C} 4$ is the middle $\mathrm{C}, 261.6 \mathrm{~Hz})$; and $y$-axis denotes the amplitude in $\mathrm{dB}$.

There is a strong tendency that the partials of 6th order and up at grade S3 do not decay monotonically, but grow at first slowly then decay undulatingly. Also their durations are longer than those at grade $\mathrm{S} 0$ as a whole.

Let $A$ be the largest among all the peak amplitudes, $A_{i}$, of the $i$-th partials $(i=1,2, \cdots, 24)$. Apparently $A=A_{6}$ for S0, as well as for S3, in Fig. 5. Now, let $d_{i}$ be the timespan of $i$-th partial when the amplitude stays above $\alpha A$, where $\alpha$ is a small fractional value. Table 5 lists $d_{i}$ 's $(i=1,2, \cdots, 24)$ of S0 and S3 for $\alpha=10^{-2}$. The partials of S3 have larger $d_{i}$ 's than those of S0 except at $i=3,4,22$, and 23 ; and specifically, more than twice as large as those of S0 at $8 \leq i \leq 20$ except $i=13$ and 14 .
Figure 6 shows the waveforms of the open second string, similarly to Fig. 3 for the open first string. At time section $200 \mathrm{~ms}$, the waveform at grade S0 is smooth and of smaller amplitude as well. It seems to contain hardly high-frequency partials. On the other hand, the waveform at grade S3 contains much of high-frequency partials together with the low-frequency ones. Note that the initial amplitude at grade $\mathrm{S} 0$ is larger than $\mathrm{S} 3$, but the relation is reversed after $200 \mathrm{~ms}$. Table 6 shows the temporal development of RMS (root-mean-square) value of waveforms $\mathrm{S} 0$ and $\mathrm{S} 3$ in the unit of $\mathrm{dB}$, similarly to Table 3. The result implies that waveform S3 has a very long buildup time and a slow decay as compared to $\mathrm{S} 0$.
Table 5 Duration $d_{i}$ of $i$-th partial $(i=1, \ldots, 24), 1$ st string.

\begin{tabular}{rcc}
\hline & \multicolumn{2}{c}{$d_{i}(\mathrm{~s})$} \\
\cline { 2 - 3 } & $\mathrm{S} 0$ & $\mathrm{~S} 3$ \\
\hline 1 & 0.21 & 0.23 \\
2 & 0.19 & 0.20 \\
3 & 0.49 & 0.36 \\
4 & 1.06 & 0.84 \\
5 & 1.54 & 1.55 \\
6 & 1.48 & 2.17 \\
7 & 1.03 & 1.25 \\
8 & 0.73 & 1.57 \\
9 & 0.21 & 0.53 \\
10 & 0.26 & 1.18 \\
11 & 0.17 & 1.18 \\
12 & 0.22 & 1.31 \\
13 & 0.41 & 0.79 \\
14 & 0.11 & 0.13 \\
15 & 0.05 & 1.21 \\
16 & 0.14 & 1.14 \\
17 & 0.09 & 0.71 \\
18 & 0.14 & 0.61 \\
19 & 0.07 & 0.34 \\
20 & 0.04 & 0.31 \\
21 & 0.06 & 0.31 \\
22 & 0.07 & 0.07 \\
23 & 0.07 & 0.06 \\
24 & 0.01 & 0.28 \\
\hline
\end{tabular}

Table 6 Temporal development of RMS values (dB) of waveforms S0 and S3, 2nd string.

\begin{tabular}{ccc}
\hline \multirow{2}{*}{ time $(\mathrm{s})$} & \multicolumn{2}{c}{ RMS values $(\mathrm{dB})$} \\
\cline { 2 - 3 } & S0 & S3 \\
\hline-0.09 & $*$ & -12.2 \\
-0.08 & $*$ & -14.2 \\
-0.07 & $*$ & -15.4 \\
-0.06 & $*$ & -6.9 \\
-0.05 & $*$ & -4.6 \\
-0.04 & $*$ & -3.2 \\
-0.03 & -15.5 & -2.4 \\
-0.02 & -7.7 & -0.9 \\
-0.01 & -2.1 & -0.2 \\
\hline 0.00 & 0.0 & 0.0 \\
\hline 0.20 & -12.2 & -2.0 \\
0.40 & -21.0 & -16.8 \\
0.60 & -25.7 & -15.5 \\
0.80 & -28.5 & -20.1 \\
1.00 & -35.2 & -23.7 \\
1.20 & -45.1 & -24.7 \\
1.40 & -42.1 & -24.7 \\
1.60 & -47.5 & -34.7 \\
1.80 & -49.2 & -39.8 \\
2.00 & -49.4 & -39.2 \\
\hline 2.50 & $*$ & -48.0 \\
3.00 & $*$ & -51.5 \\
\hline$*$ below $-55 \mathrm{~dB}$ & \\
& &
\end{tabular}



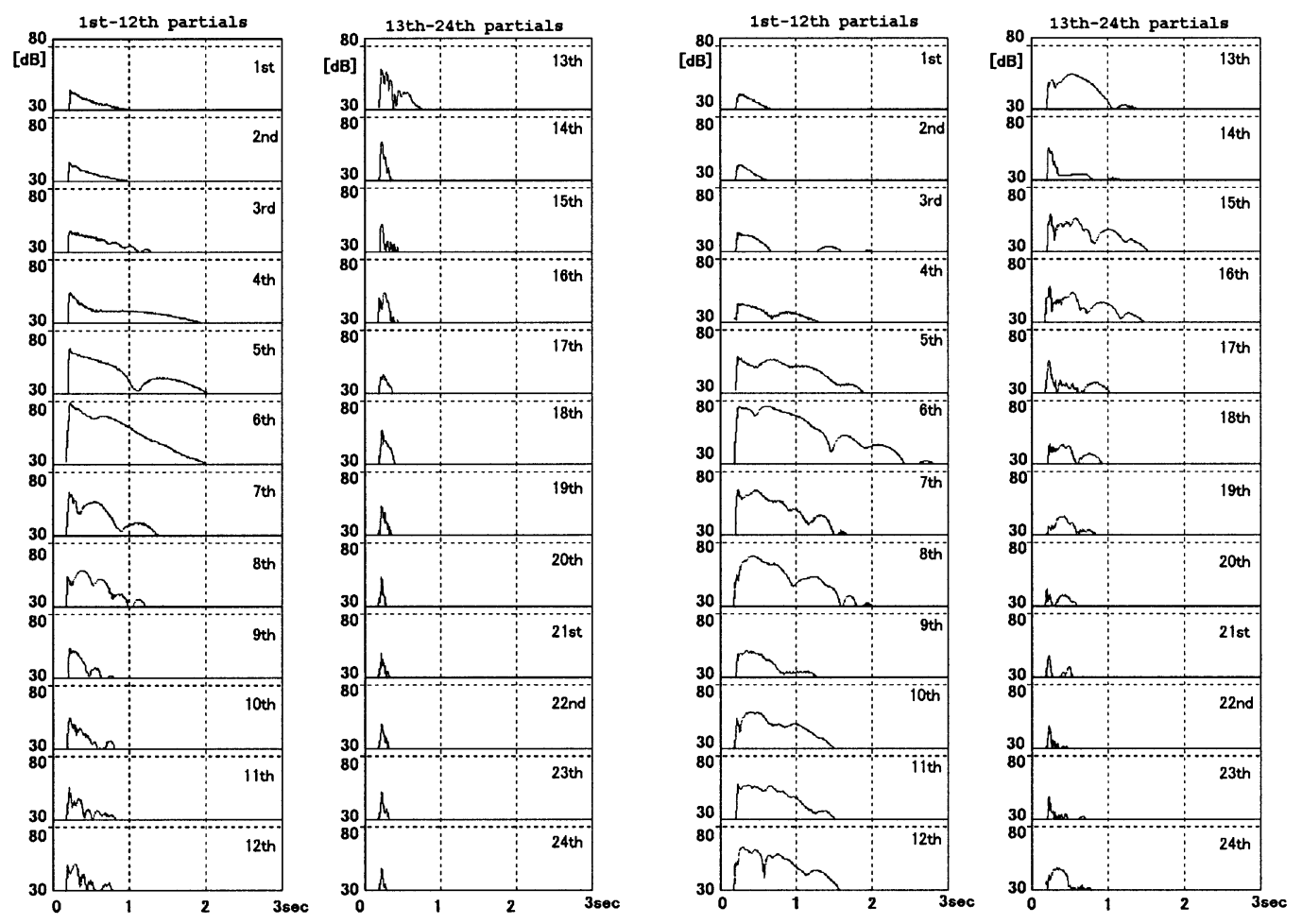

Fig. 5 Temporal development of the first 24 partials of the open first string; comparison of S0 (left) and S3 (right).

(Sawari S0)

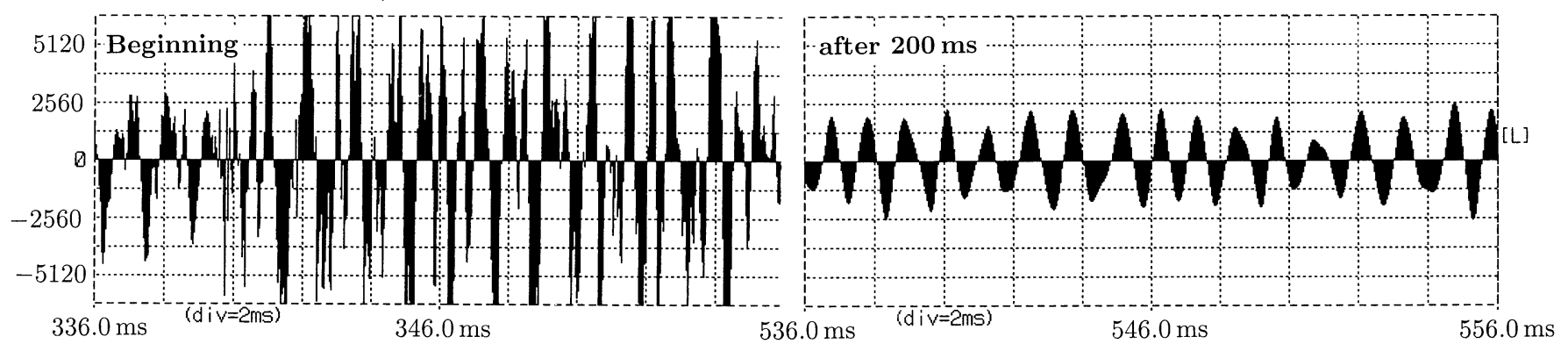

(Sawari S3)
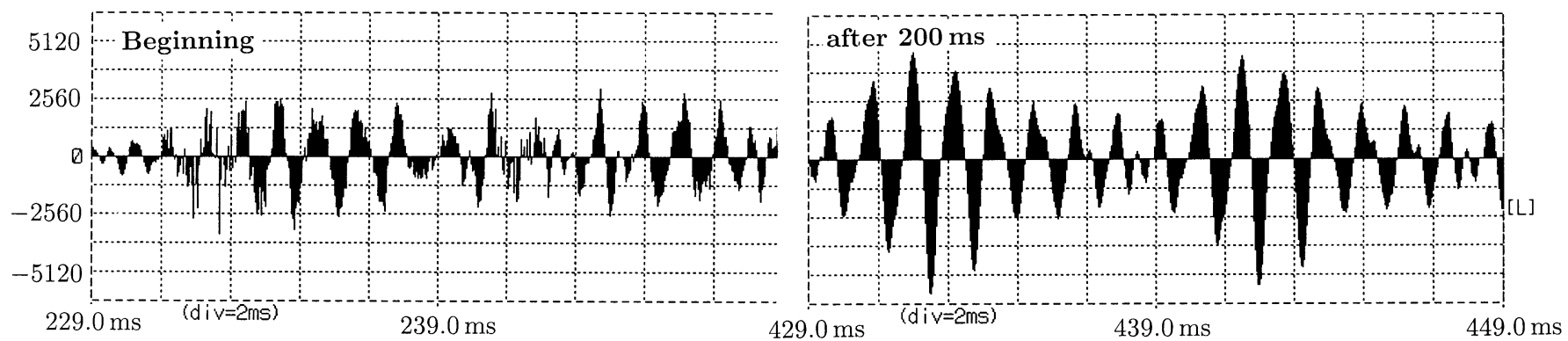

Fig. 6 Waveforms of the open second string; comparison of S0 (top) and S3 (bottom).

Figure 7 shows the power spectra at grade $\mathrm{S} 0 \sim \mathrm{S} 3$, each in the time interval of $341.3 \mathrm{~ms}$ starting at the time point when the acoustic energy becomes its peak level after onset. The partials of 8th order and up get boosted as the sawari grade proceeds from S0 to S3. Table 7 lists the spectral centroids $f_{\mathrm{c}}$ 's correponding to the power spectra
S0, S1, S2, and S3 in Fig. 7, similarly to Table 4. The $f_{\mathrm{c}}$ becomes higher as the sawari grade proceeds though not in a monotonical way as it was in the case of Fig. 3, e.g., $f_{\mathrm{c}}$ of $\mathrm{S} 3$ is $1,024.5 \mathrm{~Hz}$, which is $78.1 \mathrm{~Hz}$ as high as that of So. 


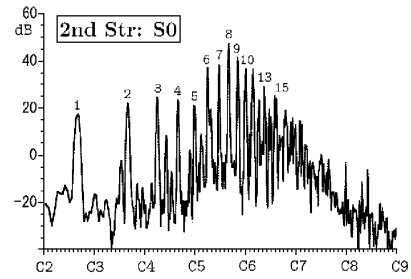

(a)

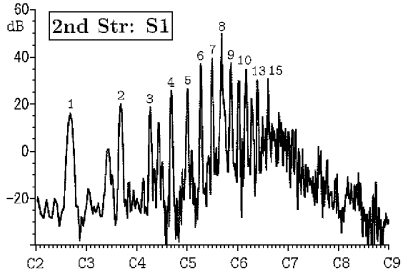

(b)

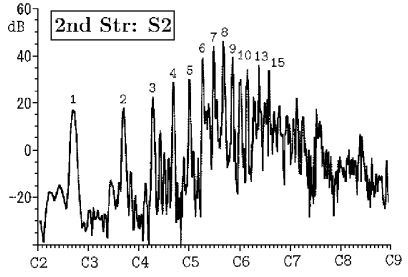

(c)

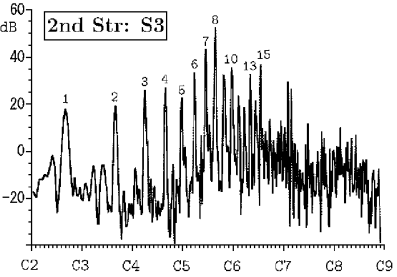

(d)

Fig. 7 Power spectra of the open second string; comparison of S0 S3. Results by DFT with Blackman's window for the interval of $341.3 \mathrm{~ms}$ (16,384 points) starting from the time when the acoustic energy reaches its peak level. The $x$-axis denotes the frequency in logarithmic scale marked with pitch names of $\mathrm{C}(\mathrm{C} 4$ is the middle $\mathrm{C}, 261.6 \mathrm{~Hz})$; and $y$-axis denotes the amplitude in $\mathrm{dB}$.
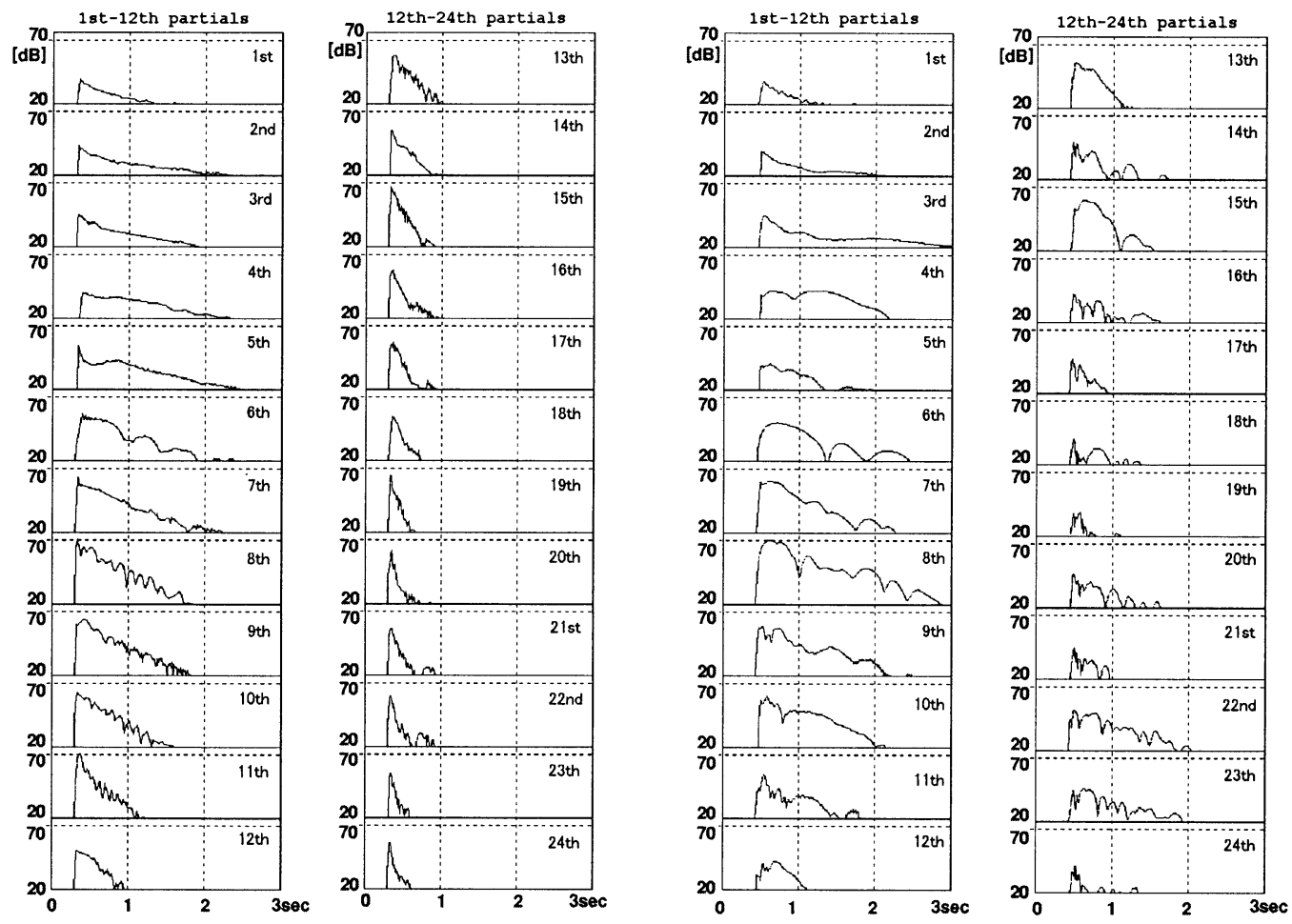

Fig. 8 Temporal development of the first 24 partials of the open second string; comparison of S0 (left) and S3 (right).

Table 7 Spectral centroid $f_{\mathrm{c}}(\mathrm{Hz}), 2$ st string.

\begin{tabular}{cccc}
\hline S0 & S1 & S2 & S3 \\
\hline 946.4 & 938.3 & $1,038.6$ & $1,024.5$ \\
\hline
\end{tabular}

Figure 8 shows the temporal development of amplitudes of up to 24th partials at grades S0 and S3. Here, the time window of 2,048 points $(41.7 \mathrm{~ms})$ was used for the open second string. In contrast to the behavior of S0 that every partial rises sharply and decays monotonically, the partials of 6th order and up at grade S3 grow at first slowly, then are going to decay undulatingly. Also their durations are longer than those at grade S0 as a whole. The 8th partial has the maximal amplitude among all the partials for both grades $\mathrm{S} 0$ and $\mathrm{S} 3$. (These two maximal values happen to be almost equal.) Table 8 lists $d_{i}$ 's of 24 partials for $\mathrm{S} 0$ and $\mathrm{S} 3$, similarly to Table 5 . The partials of S3 have longer $d_{i}$ 's than those of S0 except at $i=2,5,19$, and 24.

\section{CONCLUSION AND DISCUSSION}

The acoustical effect of sawari structure was studied experimentally for different grades of sawari, $\mathrm{S} 0 \sim \mathrm{S} 3$, by use of a Chikuzen 5-stringed biwa. Here, the grade means the strength of sawari effect determined by musical judgment, but it is related primarily to the width of strip in the sawari structure created on the top surface of shirabeguchi by shaving process. 
Table 8 Duration $d_{i}$ of $i$-th partial $(i=1, \ldots, 24), 2$ st string.

\begin{tabular}{rcc}
\hline & \multicolumn{2}{c}{$d_{i}(\mathrm{~s})$} \\
\cline { 2 - 3 } & $\mathrm{S} 0$ & $\mathrm{~S} 3$ \\
\hline 1 & 0.20 & 0.26 \\
2 & 0.46 & 0.26 \\
3 & 0.44 & 0.65 \\
4 & 0.97 & 1.38 \\
5 & 1.28 & 0.68 \\
6 & 1.06 & 1.23 \\
7 & 1.22 & 1.52 \\
8 & 1.14 & 2.16 \\
9 & 1.11 & 1.55 \\
10 & 0.94 & 1.38 \\
11 & 0.67 & 0.87 \\
12 & 0.47 & 0.51 \\
13 & 0.51 & 0.56 \\
14 & 0.34 & 0.81 \\
15 & 0.38 & 0.85 \\
16 & 0.36 & 0.42 \\
17 & 0.26 & 0.33 \\
18 & 0.21 & 0.42 \\
19 & 0.17 & 0.11 \\
20 & 0.16 & 0.59 \\
21 & 0.23 & 0.47 \\
22 & 0.42 & 1.19 \\
23 & 0.13 & 1.05 \\
24 & 0.14 & 0.11 \\
\hline & &
\end{tabular}

Analysis in the time-frequency domain has shown that the sawari effect appears in two aspects, i.e., to intensify higher partials and to elongate their durations. We have reported only the cases of the first and second open strings, but we note that the same phenomenon was found for other three open strings, though the sawari effect appeared stronger on lower strings.

The results obtained herein are certainly two physical correlates to the quality of sawari tone that the biwa player searches for as a desired biwa tone.

It is worth to cite the finding in the case of shamisen by Ando [5] that the mean decay rates become smaller (i.e., the durations become elongated) for its sawari tones than its sawari-less tones, except in the 13th partial.

Note that the results of the present paper show only the harmonic components extracted from "natural" plucked biwa tones of single open strings in the sense that the unplucked strings were left open. The sound materials might have contained components arising from the vibration of unplucked open strings due to the impact of plucking and/or sympathetic vibration. Because of this, it is impossible to study whether or not a plucked biwa tone contains inharmonic partials, i.e., partials of fractional order. Accordingly this paper did not deal with this problem.

The sawari is a nonlinear mechanism, whose physical size is very small (see Table 2), i.e., less than 1/200 of the full length of an open string (about $80 \mathrm{~cm}$ ). So it excites nonlinear vibrations whose frequency is very high; it could be in the range above 200 times the fundamental frequency of the open string. Here, the factor of 200 is based on a crude assumption on the oscillation mode with fixed boundary condition at both ends of sawari surface, i.e., above $28 \mathrm{kHz}$ for the open first string. (If one replaces the fixed boundary condition with a free one at one of the ends, the factor is reduced to 100 . Still this figure is very large.)

It is an interesting problem what the driving mechanism of such high frequency vibrations is to boost the power spectrum at middle frequency range (6th to 20th partials or thereabouts) of the resulting sawari tone. A first step to look into the actual mechanism of the sawari phenomenon must be to study the one-dimensional wave equation $\partial^{2} u / \partial t^{2}=c^{2} \partial^{2} u / \partial x^{2}(0<x<\ell, \ell=$ string length), where $u=u(t, x)$ is the displacement of the string that is subject to a unilateral, elastic constraint near one of the ends which are kept fixed. After having theoretical insights by this model, studies on suitable models of coupled string-instrumental body should be taken. All these problems are open at the present time.

\section{ACKNOWLEDGMENTS}

The authors are grateful to Ms. Kyokusumi Tohnai, player of Chikuzen biwa, for playing the instrument for our experiment. Also they appreciate Prof. Masayuki Morimoto, Kobe University, and Prof. Hideki Tachibana, Univerisity of Tokyo, for providing us with use of their acoustical facilities.

\section{REFERENCES}

[1] E. Kikkawa, "On the sawari of shamisen", in Esthetics of Shamisen and An Episode on the Birth of the Department of Japanese Music at Tokyo University of Fine Arts and Music (Shuppan Geijutsu-sha, Tokyo, 1997), pp. 113-136 (in Japanese).

[2] Y. Ando, "Survey on the instrumental structure relating to the making of Chikuzen Biwa", in Chikuzen Biwa (Technical Comittee on the Craftsmanship of Chikuzen Biwa, Fukuoka, 1977), pp. 17-22 (in Japanese).

[3] Y. Ando, "Structure of 'Chikuzen Biwas",, Ongakugaku (J. Jpn. Musicol. Soc.) 25(3), 137-152 (1979) (in Japanese).

[4] Y. Ando, "Relation among structural, acoustical and tonequalitative difference observed in some twenty ChikuzenBiwas", Ongakugaku (J. Jpn. Musicol. Soc.) 27(2), 81-97 (1981) (in Japanese).

[5] Y. Ando, Acoustics of Musical Instruments (Ongakunotomo-sha, Tokyo, 1996) (in Japanese).

[6] Y. Tohnai and K. Kishi, "Structure and examples of played waves of the satsuma biwa", Tech. Rep. Musical Acoust., Acoust. Soc. Jpn. MA93-19 (1993) (in Japanese).

[7] K. Kishi, Y. Tohnai and M. Yamada, "The "Satsuma" biwa", Proc. 3rd Jt. Meet. ASA and ASJ (Honolulu, December 1996), pp. 325-330.

[8] K. Tohnai, Study on Japanese Modern Biwas (Kasama-shoin, 
Tokyo, 1994) (in Japanese).

[9] K. Tohnai, Sequel of Study on Japanese Modern Biwas (Kasama-shoin, Tokyo, 1998) (in Japanese).

[10] Y. Takasawa, "Accurate estimation by interpolation formulae in discrete Fourier transforms", Tech. Rep. Musical Acoust., Acoust. Soc. Jpn. MA89-26 (1989) (in Japanese).

Tomoyasu Taguti was born in 1939. He is a Professor of the Faculty of Science, Konan University. He received the B.E. and M.S. degrees in Applied Physics from the University of Tokyo, and Dr. Eng. from the same Univesity. His current interests are in musical acoustics, especially in the physical and psychological aspects of music perfor- mance, and in the physics of musical instruments. He is a member of ASJ, Information Processing Society of Japan, Japanese Society of Music Perception and Cognition, and Mathematical Society of Japan.

Yoshimasa (Kakuryo) Tohnai was born in 1938. He received the M.A. degree from Nihon University in 1975 . He is a teacher of Busan High School, Nihon University. He is active in the performance of biwa music as a Satsuma-biwa player, and also in the study of the biwa instrument. He is a member of the Japan Pen Club and the Association for Popular Literature. He is now a student in the doctoral course of the Graduate School of Art, Nihon University. 